\title{
Exogenous spermine inhibits high glucose/oxidized LDL-induced oxidative stress and macrophage pyroptosis by activating the Nrf2 pathway
}

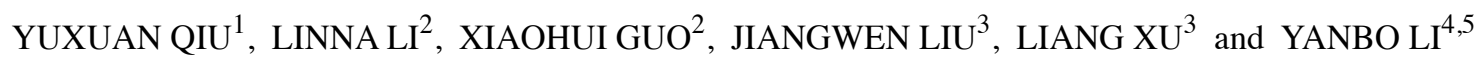 \\ ${ }^{1}$ Department of Endocrinology and Metabolic Disease, Harbin Medical University, Harbin, Heilongjiang 150076; \\ ${ }^{2}$ Department of Laboratory Medicine, Harbin City First Hospital, Harbin, Heilongjiang 150010; \\ ${ }^{3}$ Department of Endocrinology and Metabolic Disease, Southern University of Science and Technology, \\ Shenzhen, Guangdong 518055; ${ }^{4}$ Department of Endocrinology and Metabolic Disease, \\ The First Affiliated Hospital of Harbin Medical University, Harbin, Heilongjiang 150007; \\ ${ }^{5}$ Department of Endocrinology, South China Hospital Affiliated to Shenzhen University, \\ Shenzhen, Guangdong 518061, P.R. China
}

Received December 7, 2021; Accepted February 2, 2022

DOI: $10.3892 / \mathrm{etm} .2022 .11239$

\begin{abstract}
Evidence suggests that macrophage pyroptosis promotes the progression of diabetic atherosclerosis. Spermine, a natural cellular metabolite, demonstrates a protective effect against cardiovascular diseases. However, whether spermine has a protective effect against macrophage pyroptosis caused by high glucose (HG) and oxidized low-density lipoprotein (ox-LDL) conditions remains to be elucidated. To investigate the protective effect of spermine and the related underlying mechanism, THP-1 macrophages were treated with HG/ox-LDL, spermine, or the specific nuclear factor erythroid 2-related factor 2 (Nrf2) inhibitor ML385. Cell viability was detected using CCK-8, cell membrane permeability was analyzed using lactate dehydrogenase (LDH) and Hoechst/propidium iodide staining and pyroptosis-related gene and protein expression levels were evaluated using polymerase chain reaction and western blot analysis. Spermine showed a potent preventive effect on THP-1 macrophage pyroptosis and oxidative stress induced by HG/ox-LDL. Cells treated with spermine showed increased cell viability, reduced reactive oxygen species (ROS) production, decreased LDH levels in the supernatant and reduced cell swelling. In addition, spermine significantly reduced NLR family pyrin domain containing 3, cleaved caspase-1, N-gasdermin D and IL-1 $\beta$ expression, as well as IL-1 $\beta$ levels in the supernatant. This
\end{abstract}

Correspondence to: Dr Yanbo Li, Department of Endocrinology and Metabolic Disease, The First Affiliated Hospital of Harbin Medical University, 23 Post Office Street, Nangang, Harbin, Heilongjiang 150007, P.R. China

E-mail: lybhmu2019@163.com

Key words: THP-1 cells, reactive oxygen species, diabetic atherosclerosis demonstrated that the inhibition of pyroptosis and oxidative stress due to spermine was Nrf2 dependent. Furthermore, spermine enhanced Nrf2 nuclear translocation, thereby increasing heme oxygenase-1 and NADPH quinone oxidoreductase-1 expression, which subsequently reduced ROS production. In addition, the anti-pyroptotic and antioxidant effects of spermine were reversed by ML385 inhibition of Nrf2. It was concluded that spermine prevented macrophage pyroptosis and increased ROS overproduction by activating the Nrf2 pathway. The data suggested that spermine may be a potential novel drug for the treatment of diabetic atherosclerosis because it targets macrophage pyroptosis.

\section{Introduction}

Atherosclerosis is a chronic inflammatory arterial angiopathy that is a common cause of cardiovascular disease (1). There is considerable evidence demonstrating that diabetes significantly exacerbates the progression of atherosclerosis (2). Blood glucose and lipid metabolism disorders are among the commonest pathophysiological mechanisms of diabetic atherosclerosis (3). It has been found that macrophage dysfunction contributes to the development of atherosclerosis. This dysfunction is characterized by an increased inflammatory response and problems with phagocytes, which ultimately increase the likelihood of plaque rupture (4). A large number of inflammatory factors, including IL-1 $\beta$ and IL-18, are released from pyroptotic macrophages and contribute to the instability of atherosclerotic plaques in diabetes (5). It is also reported that sinapic acid attenuates diabetic atherosclerosis by suppressing macrophage pyroptosis (6). Therefore, preventing macrophage death may be an effective method for preventing diabetic atherosclerosis. Pyroptosis is a form of proinflammatory programmed cell death that requires NLR family pyrin domain containing 3 (NLRP3) inflammasome activation and mutation of gasdermin D (GSDMD) (7). Interaction of NLRP3 with apoptosis-associated speck-like protein (ASC) 
containing a caspase recruitment domain (CARD) causes recruitment of caspase-1. This triggers the cleavage and release of IL- $1 \beta$ through pores formed by N-GSDMD (8). Macrophage pyroptosis serves an important role in the worsening of atherosclerotic plaques (9). NLRP3 and caspase-1 gene deletions significantly slow the progression of arteriosclerosis (10). Thus, it is of great significance to identify drugs that are protective against pyroptosis in order to prevent and treat diabetic atherosclerosis.

Oxidative stress is considered a potential agonist of inflammasome activation. Previous studies have shown that excessive production of reactive oxygen species (ROS) can activate NLRP3 inflammasome and lead to pyroptosis $(7,11)$. Nuclear factor erythroid 2-related factor 2 (Nrf2) is a common transcription factor that exerts antioxidant, anti-apoptotic and anti-inflammatory effects by interacting with multiple promoters (12). When increased oxidative stress is sensed, $\mathrm{Nrf} 2$ translocates into the nucleus and promotes the transcription of antioxidant proteins (13). Heme oxygenase-1 (HO-1) and NADPH quinone oxidoreductase-1 (NQO-1) are important antioxidant proteins downstream of $\mathrm{Nrf} 2$, which inhibit oxidative stress-induced pyroptosis (14). In a diabetic atherosclerosis model, exogenous hydrogen sulfide stimulates the Nrf2/HO-1 pathway, thus improving macrophage function and inhibiting atherosclerosis progression (15). In addition, pharmacological activation of $\mathrm{Nrf} 2$ suppresses endothelial pyroptosis by reducing the overproduction of ROS (16). It is reported that Nrf2 may be an ideal therapeutic target for prevention of diabetic atherosclerosis (17).

Spermine is a small polyamine that is a natural product of cellular metabolism (18). It is reported that spermine is involved in regulating cell proliferation, apoptosis and inflammation (19). Notably, metabolomic analysis shows that problems with spermine metabolism can lead to a decrease in spermine levels, which is often observed in atherosclerotic plaques (20). Furthermore, spermine can directly activate the Nrf2 pathway to attenuate cardiomyopathy in diabetic rats (21). However, it is unclear whether spermine activation of the Nrf2 pathway can inhibit macrophage pyroptosis.

The present study investigated whether spermine could attenuate macrophage pyroptosis under high glucose (HG) and oxidized low-density lipoprotein (ox-LDL) conditions. Furthermore, it explored the underlying mechanism by which spermine prevented macrophage pyroptosis and investigated its ability to suppress ROS production via Nrf2 activation.

\section{Materials and methods}

Reagents. Human monocytic THP-1 cells were obtained from American Type Culture Collection. Spermine was purchased from MilliporeSigma. Ox-LDL was purchased from Guangzhou Yiyuan Biological Technology Co. Ltd. Phorbol 12-myristate 13-acetate (PMA), DCFDA Assay kit, lactate dehydrogenase (LDH) Assay kit, Lipid Peroxidation MDA Assay kit, GSH and GSSG Assay kit and Hoechst/PI Staining kit were obtained from Beyotime Institute of Biotechnology. The CCK-8 kit was obtained from Dojindo Laboratories, Inc. SYBR Green and reverse transcription kits were obtained from Roche Diagnostics. Primary antibodies against NLRP3 (cat. no. ab263899), N-GSDMD (cat. no. ab210070), cleaved caspase-1 (cat. no. ab179515), Nrf2 (cat. no. ab137550), HO-1 (cat. no. ab52947), NQO-1 (cat. no. ab28947) and IL-1 $\beta$ (cat.no.ab254360) were purchased from Abcam. The secondary antibody was HRP-labeled Goat Anti-Rabbit IgG (H+L) (cat. no. A0208), purchased from Beyotime Institute of Biotechnology.

Cell culture and induction. THP-1 cells were cultured in RPMI-1640 medium (Gibco; Thermo Fisher Scientific, Inc.) supplemented with $10 \%$ fetal bovine serum (Gibco; Thermo Fisher Scientific, Inc.). Cell culture medium was replaced every 2 days and the cultures were maintained at $37^{\circ} \mathrm{C}, 5 \% \mathrm{CO}_{2}$ in a fully humidified incubator. THP-1 cells were induced into macrophages using PMA pretreatment (50 nM PMA for $48 \mathrm{~h}$ before the experiment at $37^{\circ} \mathrm{C}$ ). The cells were then divided into the following groups: The control group, which was treated with phosphate-balanced saline (PBS) or dimethyl sulfoxide; the ox-LDL/HG group, which was treated with D-glucose (D-Glu, $25 \mathrm{mmol} / \mathrm{l}$ ) and ox-LDL $(100 \mu \mathrm{g} / \mathrm{ml})(15,22)$; the spermine group, which was treated with spermine $(5 \mu \mathrm{mol} / \mathrm{l})$, D-Glu $(25 \mathrm{mmol} / \mathrm{l})$ and ox-LDL $(100 \mathrm{ng} / \mathrm{ml})$; and the ML385 group, which was treated with NRF2 inhibitor ML385 (5 $\mu \mathrm{mol} / \mathrm{l})$, spermine ( $5 \mu \mathrm{mol} / \mathrm{l})$, D-Glu $(25 \mathrm{mmol} / \mathrm{l})$ and ox-LDL $(100 \mathrm{ng} / \mathrm{ml})$.

Enzyme-linked immunosorbent assay (ELISA). The cell supernatant of the macrophages was collected and the levels of IL-1 $\beta$ were determined using their corresponding ELISA kit (cat. no. EK0392; Wuhan Boster Biological Technology, Ltd.) according to the manufacturer's protocols.

Scanning electron microscopy (SEM). Macrophages were planted in glass sliders, and the cells were divided into experimental groups. Cells were immobilized at room temperature with glutaraldehyde $(2.5 \%)$ for $48 \mathrm{~h}$. The sample was rinsed with acetone and immersed in iso-amyl acetate and then exposed to air at room temperature for dehydration for $\sim 3 \mathrm{~h}$. Finally, gold palladium film was added to the sample using a vacuum plating method and observed under scanning electron microscope at x3,000 magnification.

Cell viability assays. Macrophages were cultured in 96-well plates with 10,000 cells per well. The CCK- 8 assays were performed according to the manufacturer's instructions. After preparing the experimental groups and performing the cell treatments, a 1:10 dilution of the CCK-8 working solution was prepared. Then, $100 \mu 1$ working solution was removed and added to 96-well plates. The plates were placed in an incubator at $37^{\circ} \mathrm{C}$ for $60 \mathrm{~min}$ to allow the reaction to occur. After the incubation, absorbance was measured at $450 \mathrm{~nm}$ using a microplate spectrophotometer (Tecan Group, Ltd.).

LDH assay. Macrophages were cultured in 96-well plates with 10,000 cells per well. According to the LDH Assay kit manufacturer's instructions, the cell culture plates were centrifuged at $400 \mathrm{x}$ g for 5 min using a multi-well plate centrifuge following drug stimulation. The LDH-releasing reagent provided in the kit was diluted 10 -fold with PBS and mixed well. The cell supernatant was aspirated and $150 \mu 1$ of the diluted LDH-releasing reagent was added to each cell sample. 
The plates were shaken to assure that the LDH-releasing reagent in each well was mixed well. The plates were then incubated at $37^{\circ} \mathrm{C}$ for $1 \mathrm{~h}$. The cell culture plates were subsequently centrifuged for $5 \mathrm{~min}$ at $400 \mathrm{x} \mathrm{g}$ in a multi-well plate centrifuge. Thereafter, $120 \mu \mathrm{l}$ supernatant was collected from each well and separately added to the corresponding wells of a new 96-well plate. subsequently, the absorbance was measured at $490 \mathrm{~nm}$ using a microplate spectrophotometer.

Reverse transcription-quantitative (RT-q) PCR. A total of $1 \times 10^{6}$ cells $/ \mathrm{ml}$ were seeded in 6 -well plates for RNA extraction (23). Total RNA was isolated from the macrophages using TRIzol ${ }^{\circledR}$ reagent (Invitrogen; Thermo Fisher Scientific, Inc.) according to the manufacturer's instructions. RNA concentration was determined using a spectrophotometer (BioSpec-nano; Shimadzu Corporation) to measure absorbance at $260 \mathrm{~nm}$. Complementary DNA (cDNA) was synthesized using the RNA as template using a Reverse Transcription kit (Roche Diagnostics). qPCR was then performed using SYBR Green (Roche Diagnostics). Each reaction was performed in triplicate. The cycling profile was as follows: Initial denaturation at $95^{\circ} \mathrm{C}$ for $5 \mathrm{~min}$, followed by 40 cycles of denaturation at $94^{\circ} \mathrm{C}$ for $10 \mathrm{sec}$, annealing at temperatures specific for each pair of primers (ranging from 53 to $62^{\circ} \mathrm{C}$ ) for $10 \mathrm{sec}$, extension at $72^{\circ} \mathrm{C}$ for $28 \mathrm{sec}$ and a final elongation at $72^{\circ} \mathrm{C}$ for $5 \mathrm{~min}$. A melt-curve analysis was performed to quantify the PCR products. The primer sequences for the target genes used in this study was shown in Table SI. All data were normalized to $\beta$-actin mRNA levels.

Hoechst/propidium iodide (PI) staining. Cells were seeded into 24-well plates with 50,000 cells per well. After the cells were treated according to the experimental design, the cells were washed three times with PBS. Then, $5 \mu 1$ Hoechst staining solution was added, followed by $5 \mu \mathrm{l}$ PI stain. The cells were mixed and incubated in an ice bath at $4^{\circ} \mathrm{C}$ for $20-30 \mathrm{~min}$. The cells were carefully washed three times with PBS and an anti-fluorescence quenching agent (Beyotime Institute of Biotechnology) was added. Cells were observed under a fluorescence microscope at x200 magnification and images captured. To calculate the percentage of PI-positive cells, the number of PI-positive cells was divided by the number of Hoechst-positive cells. Three random fields were chosen for this calculation.

Western blot analysis. Following treatment, the cells were washed once with pre-cooled PBS. Radioimmunoprecipitation assay buffer containing phenylmethylsulfonyl fluoride was added to fully lyse the cells. The lysate was collected and placed on ice for $30 \mathrm{~min}$. The lysate was then centrifuged at $14,000 \mathrm{x}$ g at $4^{\circ} \mathrm{C}$ for $15 \mathrm{~min}$ to collect the supernatant. The concentration of the protein in the macrophage lysates was detected using a BCA kit (Beijing Solarbio Science \& Technology Co., Ltd.). Aliquots of each sample containing $30 \mu \mathrm{g}$ of protein were prepared and loading buffer was added to these. The proteins were then separated using $10 \%$ SDS-polyacrylamide gel electrophoresis. The separated proteins were transferred onto $0.22-\mu \mathrm{m}$ polyvinylidene fluoride membranes (MilliporeSigma). The PVDF membranes were blocked with $5 \%$ skimmed milk at room temperature for
$1 \mathrm{~h}$ to prevent non-specific antibody binding and then washed three times with PBS. The PVDF membranes were then incubated with the indicated primary antibody for $12 \mathrm{~h}$ at $4^{\circ} \mathrm{C}$. The membranes were then washed three times with PBS. The appropriate secondary antibody (HRP-labeled Goat Anti-Rabbit IgG; $1: 10,000)$ was added to the membrane, followed by a 1-h incubation at room temperature. The membranes were washed three times at room temperature for $1 \mathrm{~h}$ each. The protein bands were visualized using an enhanced chemiluminescence reagent (Beijing Solarbio Science \& Technology Co., Ltd.). Image J software v. 1.4 (National Institutes of Health) was used to analyze the gray level intensity of the blot strips.

Total ROS measurements. Macrophages were cultured in 96-well plates with 10,000 cells per well. Macrophages were treated according to the experimental design. 2',7'-Dichlorodihydrofluorescein diacetate (DCFH-DA) was diluted 1:1,000 with serum-free medium to obtain a final concentration of $10 \mathrm{mM}$. The cell culture medium was removed, $100 \mu 1$ diluted DCFH-DA was added and the cells were incubated for $20 \mathrm{~min}$ at $37^{\circ} \mathrm{C}$. The cells were then washed three times with serum-free cell culture medium to fully remove any DCFH-DA that had not entered the cells. The cells were then subjected to fluorescence spectrophotometric analysis, as well as fluorescence microscopy at x200 magnification using an excitation wavelength of $488 \mathrm{~nm}$ and emission wavelength of $525 \mathrm{~nm}$.

Immunofluorescent staining. Macrophages were seeded into 24-well plates, treated with $4 \%$ paraformaldehyde for $10 \mathrm{~min}$ at room temperature, washed three times with PBS and then treated with $0.3 \%$ Triton for 5 min to disrupt the cell membrane at room temperature. The cells were then washed three times with PBS and then $5 \%$ bovine serum albumin was added followed by incubation for $60 \mathrm{~min}$ to reduce non-specific staining at room temperature. After washing the samples three times with PBS, anti-Nrf2 primary antibody (1:100) was added and the mixture was left to incubate overnight at $4^{\circ} \mathrm{C}$. The secondary antibody (1:400) was added and the samples were incubated at room temperature for $1 \mathrm{~h}$ at room temperature. To stain the nuclei, the cells were incubated with 4',6-diamidino-2-phenylindole (DAPI) for $5 \mathrm{~min}$ at room temperature. The cells were washed three times with PBS and then observed using a fluorescence microscope at x400 magnification.

Statistical analysis. The data were collected from three independent experiments and all results are presented as the mean \pm SD. Differences between two or more groups were estimated using unpaired Student's t-test or one-way ANOVA followed by Tukey's post hoc test, respectively. The statistical significance of the data was determined using analysis of variance with the SPSS v22.0 software (IBM Corp.). $\mathrm{P}<0.05$ was considered to indicate a statistically significant difference.

\section{Results}

Spermine prevented macrophage pyroptosis induced by $H G / o x-L D L$. The results of CCK-8 assay showed that $<5 \mu \mathrm{M}$ spermine had no significant cytotoxicity on THP-1 
A
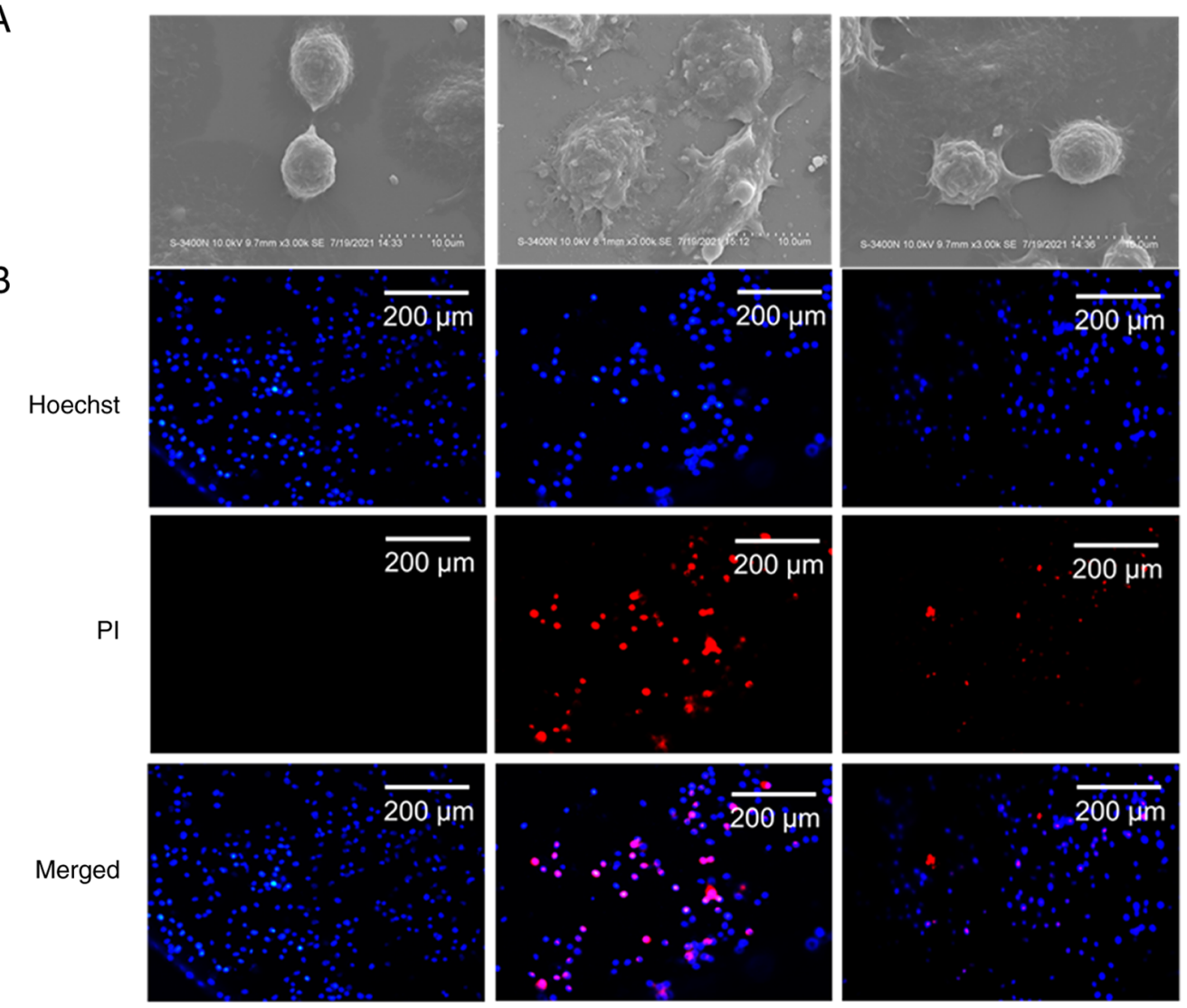

HG/ox-LDL

Spermine

$+$

$+$

C

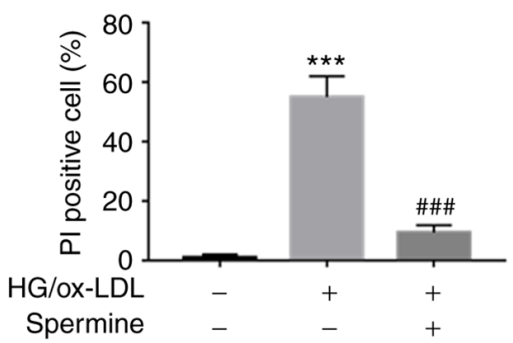

D

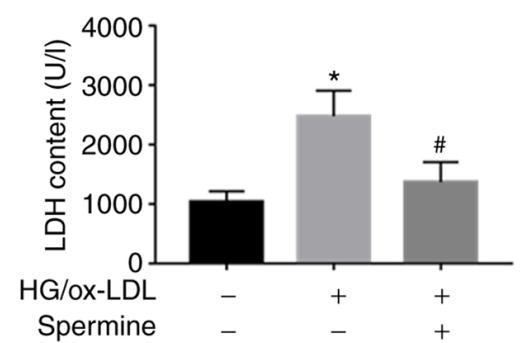

E

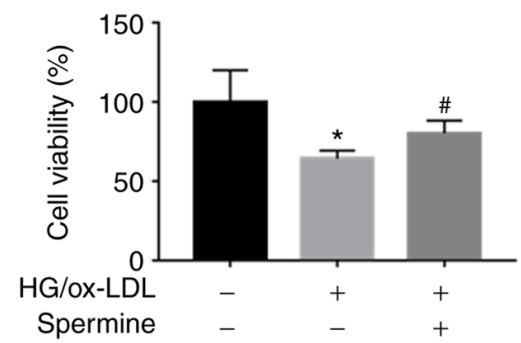

Figure 1. Spermine inhibits macrophages pyroptosis. Macrophages were divided into three groups, control group (PBS), HG/ox-LDL group (25 mmol/1 D-Glu and $100 \mu \mathrm{g} / \mathrm{ml}$ ox-LDL treatment for $24 \mathrm{~h}$ ) and Spermine group ( $5 \mu \mathrm{M}$ spermine pretreatment for 30 min followed by the addition of $25 \mathrm{mmol} / 1$ D-Glu and $100 \mu \mathrm{g} / \mathrm{ml}$ ox-LDL treatment for $24 \mathrm{~h}$ ). (A) Scanning electron microscopy was used to detect the morphological changes of macrophages. (B and C) Hoechst/PI was used to detect cell membrane permeability. (D) The LDH kit was used to detect LDH levels in supernatant. (E) CCK-8 was used to measure

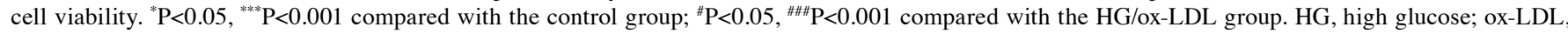
oxidized low-density lipoprotein; D-Glu, D-glucose; PI, propidium iodide.

macrophages (Fig. S1A). Cell swelling and cell membrane rupture are common characteristics of pyroptosis. To determine the effect of spermine on HG/ox-LDL-induced pyroptosis in macrophages, THP-1 derived macrophages were treated with D-glucose $(25 \mathrm{mmol} / \mathrm{l})$ and ox-LDL $(100 \mathrm{ng} / \mathrm{ml})$ for $24 \mathrm{~h}$ in the absence or presence of spermine $(5 \mu \mathrm{mol} / 1)$ at $37^{\circ} \mathrm{C}$. SEM was used to detect changes in macrophage morphology, PI staining and LDH assays were used to evaluate cell membrane integrity and CCK-8 assays were performed to determine cell viability. Macrophages swelled and enlarged in response to HG/ox-LDL stimulation and spermine pretreatment reversed these effects (Fig. 1A). PI staining revealed that spermine decreased the percentage of PI-positive macrophages in the
HG/ox-LDL group (Fig. 1B and C). In addition, it was found that HG/ox-LDL treatment led to increased LDH levels in the supernatant. However, spermine administration markedly decreased the LDH levels in HG/ox-LDL-treated macrophages (Fig. 1D), indicating a decrease in the rates of cell swelling and membrane rupture. The CCK- 8 assays showed that spermine pretreatment significantly reversed the decrease in cell viability caused by HG/ox-LDL conditions (Fig. 1E). These data suggested that spermine inhibited HG/ox-LDL-induced macrophage pyroptosis.

Spermine suppressed macrophage pyroptosis by inhibiting NLRP3 inflammasome activation. Activation of the NLRP3 


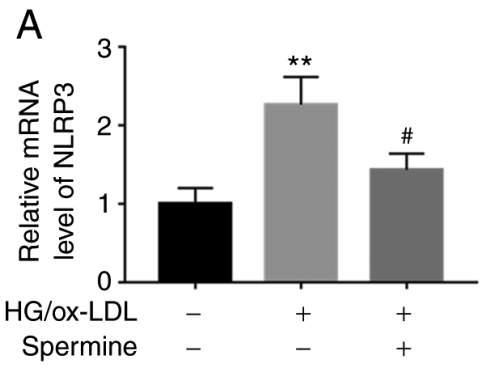

D

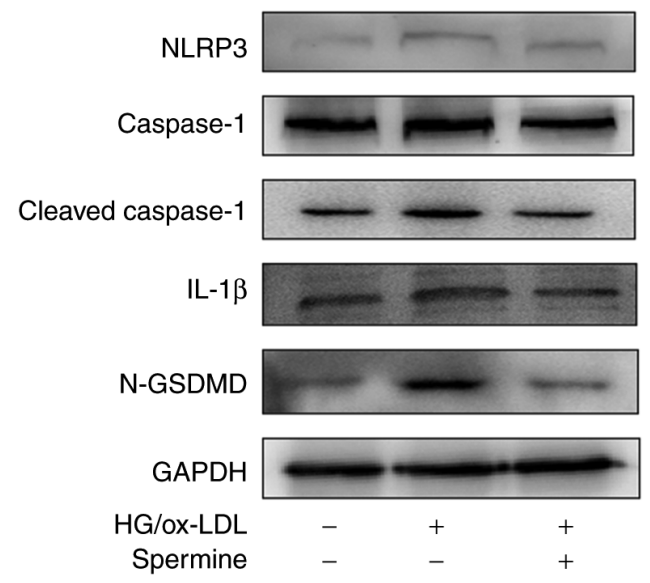

B

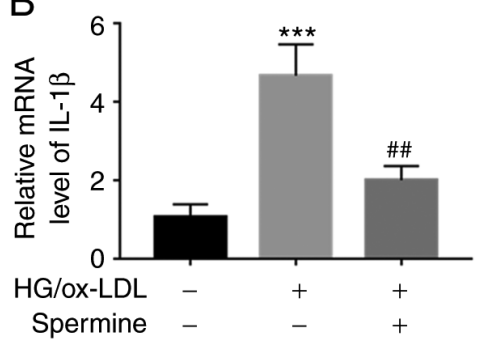

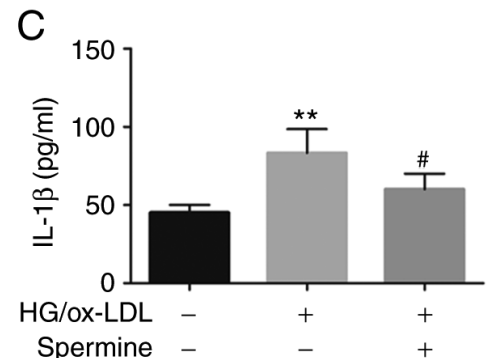

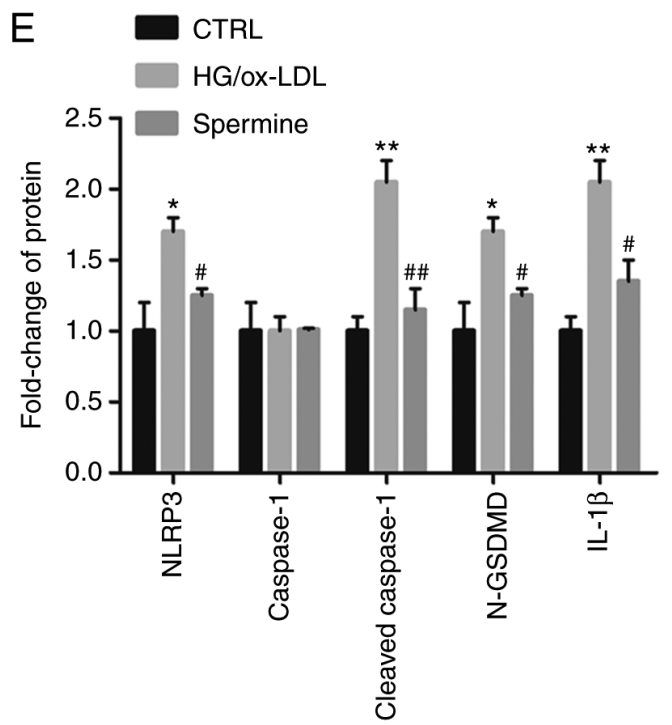

Figure 2. Spermine suppresses NLRP3 inflammasome activation under HG/ox-LDL condition. PCR was used to detect NLRP3 and IL-1 $\beta$ mRNA levels in macrophages. ELISA was used to detect IL-1 $\beta$ levels in cell supernatant. Western blotting was used to detect the levels of NLRP3, caspase-1, cleaved-caspase-1, IL-1 $\beta$ and N-GSDMD in lysate. GAPDH was the internal reference. (A) NLRP3 mRNA level. (B) IL-1 $\beta$ mRNA level. (C) IL-1 $\beta$ level in supernatant. (D) Protein level of NLRP3, caspase-1, cleaved-caspase-1, IL-1 $\beta$ and N-GSDMD in control, HG/ox-LDL and spermine group. (E) Quantification of NLRP3, caspase-1, cleaved-caspase-1, IL-1 $\beta$ and N-GSDMD. ${ }^{*} \mathrm{P}<0.05,{ }^{* *} \mathrm{P}<0.01,{ }^{* * * *} \mathrm{P}<0.001$ compared with the control group; ${ }^{\#} \mathrm{P}<0.05,{ }^{\# \#} \mathrm{P}<0.01$ compared with the HG/ox-LDL group. NLRP3, NLR family pyrin domain containing 3; HG, high glucose; ox-LDL, oxidized low-density lipoprotein; GSDMD, gasdermin D.

inflammasome leads to IL-1 $\beta$ expression and the mutation of GSDMD (N-GSDMD), which is cleaved by cleaved caspase-1. It was hypothesized that spermine pretreatment would suppress NLRP3 inflammasome activation. To this end, PCR and western blot analyses were performed to evaluate NLRP3, caspase-1, cleaved caspase-1, N-GSDMD and IL-1 $\beta$ expression levels. As expected, the levels of NLRP3 and IL-1 $\beta$ mRNAs were downregulated in the macrophages of the spermine group compared with that of the macrophages in the HG/ox-LDL group, which did not undergo spermine pretreatment (Fig. 2A and B). There are no significantly differences of caspase-1 and GSDMD mRNA level in these three groups (Fig. S2A and B). Furthermore, it was found that the increase in NLRP3, cleaved caspase-1, IL-1 $\beta$ and GSDMD expression in the HG/ox-LDL group was suppressed by spermine pretreatment (Fig. 2D and E). However, spermine treatment alone did not affect the expression level of cleaved caspase-1 and GSDMD in macrophages (Fig. S1B and C). IL-1 $\beta$ levels in the cell supernatant were also evaluated using ELISA and the results were consistent with those of the western blot analysis. IL-1 $\beta$ levels were measured in the HG/ox-LDL group and compared with those of the control group and it was found that spermine blocked the upregulated expression of IL-1 $\beta$ (Fig. 2C).

Spermine enhanced Nrf2 nuclear translocation and promoted transcription of antioxidant proteins. It is well established that
Nrf2 serves an important role in pyroptosis. Therefore, it was explored whether spermine could activate Nrf2 and its downstream antioxidant proteins. First, nuclear lysates were extracted from the different experimental groups and Nrf2 levels in the lysates were evaluated using western blotting. The results showed that the level of Nrf2 in the nucleus was significantly lower in the HG/ox-LDL group than in the control group, but spermine pretreatment caused an increase in nuclear Nrf2 levels (Fig. 3A). Furthermore, spermine treatment reduced NRF2 expression in cytoplasm, suggesting that spermine promoted NRF2 translocation from the cytoplasm to the nucleus (Fig. 3A). Second, levels of HO-1 and NQO-1, which are common antioxidant proteins downstream of $\mathrm{Nrf2}$, were measured in the experimental groups of macrophages. It was found that there were higher levels of HO-1 and NQO-1 in the total lysates of the HG/ox-LDL treatment group than in the lysates of the control group. Notably, this effect was significantly amplified in the spermine group (Fig. 3B and C). Third, immunofluorescence was used to visualize the cellular localization of Nrf2. The results demonstrated that spermine increased localization of Nrf2 to the nucleus (Fig. 3D and E). These results indicate that spermine promotes Nrf 2 activation by enhancing Nrf 2 nuclear translocation. macrophages via the Nrf2 pathway. Previous studies have 
A

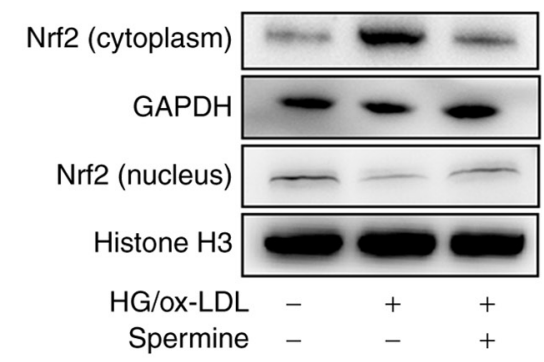

B

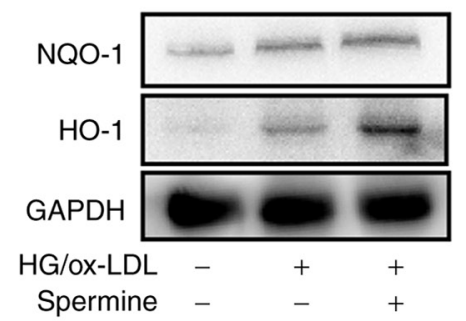

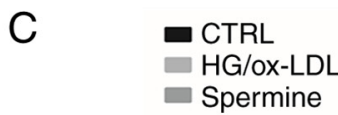

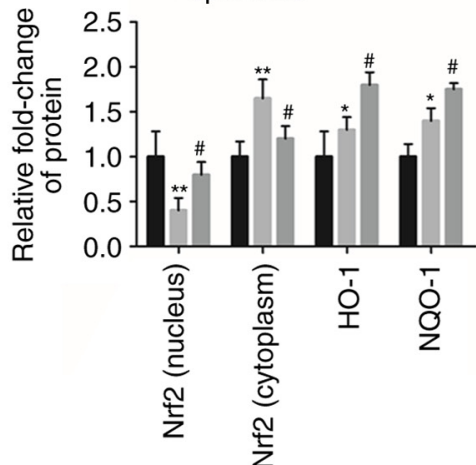

$E$
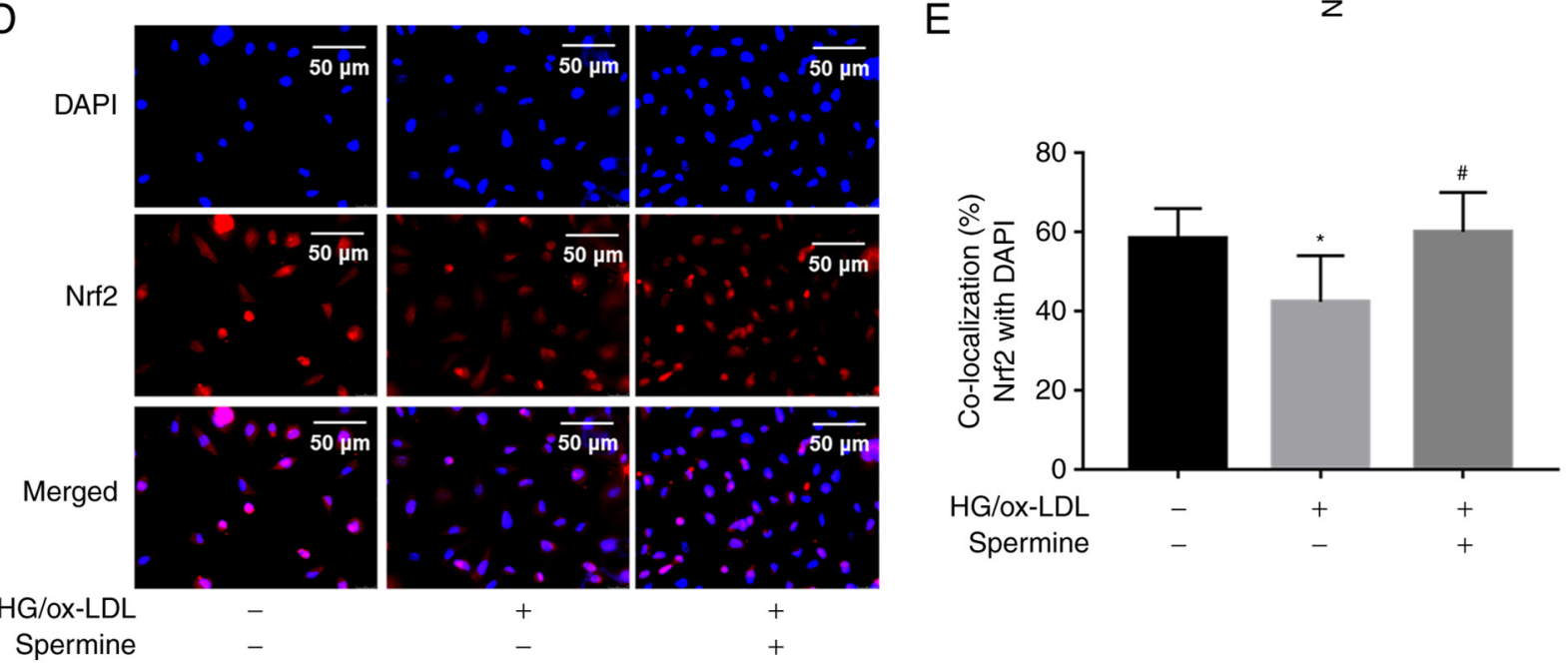

Figure 3. Spermine promotes Nrf2 to enter the nucleus and promotes antioxidant protein expression. Western blotting was used to detect the levels of Nrf2, HO-1 and NQO-1, Histone H3 and GAPDH were used as internal references for nuclear and cell total protein, respectively. Immunofluorescence was used to detect Nrf2 nuclear translocation. (A) Nrf2 level in nuclear and cytoplasm lysates. (B) HO-1 and NQO-1 level in total lysates. (C) The levels of Nrf2, HO-1 and NQO-1 were quantified. (D and E) Immunofluorescence was used to detect Nrf2 cell localization. ${ }^{*} \mathrm{P}<0.05,{ }^{* *} \mathrm{P}<0.01$ compared with the control group; ${ }^{\#} \mathrm{P}<0.05$ compared with the HG/ox-LDL group. Nrf2, nuclear factor erythroid 2-related factor 2; HO-1, heme oxygenase-1; NQO-1, NADPH quinone oxidoreductase-1; HG, high glucose; ox-LDL, oxidized low-density lipoprotein.

provided robust evidence to support the hypothesis that excessive ROS contribute to NLRP3 inflammasome activation and pyroptosis $(11,24)$. The present study aimed to confirm that spermine could reduce ROS production and investigate whether this reduction occurs via the Nrf2 pathway. The macrophages in the different experimental groups were pretreated with ML385, a specific inhibitor of Nrf2. The levels of ROS were detected in the different groups using DCFH-DA. As shown in Fig. 4A-C, ROS production and malondialdehyde (MDA) levels, a major marker of lipid peroxidation, were decreased in the spermine pretreatment group compared with those in the HG/ox-LDL group. In addition, ML385 completely abolished the anti-ROS effect of spermine. ML385 increased oxidative levels after HG/ OX-LDL treatment, however, ML385 alone had no significant effect. Furthermore, the expression of Nrf2 downstream proteins were evaluated. Analysis of glutathione (GSH) and superoxide dismutase (SOD) levels in the cell lysates revealed that spermine pretreatment resulted in increased levels of both GSH and SOD expression in macrophages subjected to HG/ox-LDL treatment and this was reversed by ML385. ML385 combined with HG/ ox-LDL treatment further reduced SOD and GSH levels, but ML385 alone did not affect SOD and GSH levels (Fig. 4D and E). In addition, western blotting was used to evaluate the protein expression levels of HO-1 and NQO-1. The findings revealed that ML385 attenuated the spermine-induced increases in HO-1 and NQO-1 expression in HG/ox-LDL-treated macrophages. ML385 attenuated the upregulation of HO-1 and NQO-1 induced by ox-LDL, but treatment with ML385 alone did not affect HO-1 and NQO-1 levels (Fig. 4F and G).

The anti-pyroptosis effect of spermine on macrophages required Nrf 2 activation. The experimental groups of cells were pretreated with ML385 to further explore whether spermine inhibited macrophage pyroptosis by stimulating the Nrf2 pathway and to investigate the mechanism underlying this process. Results from the CCK- 8 assays demonstrated that the increase in cell viability caused by spermine pretreatment was completely abolished by ML385 treatment (Fig. 5A). The LDH assays showed a significant increase in LDH levels following ML385 and HG/ox-LDL treatment without spermine (Fig. 5B). Spermine reduced the HG/ox-LDL-induced increase in the percentage of PI-positive cells, which was also reversed by ML385 treatment (Fig. 5C and D). These findings suggest that ML385 abolished the anti-pyroptosis effect of spermine. 
A
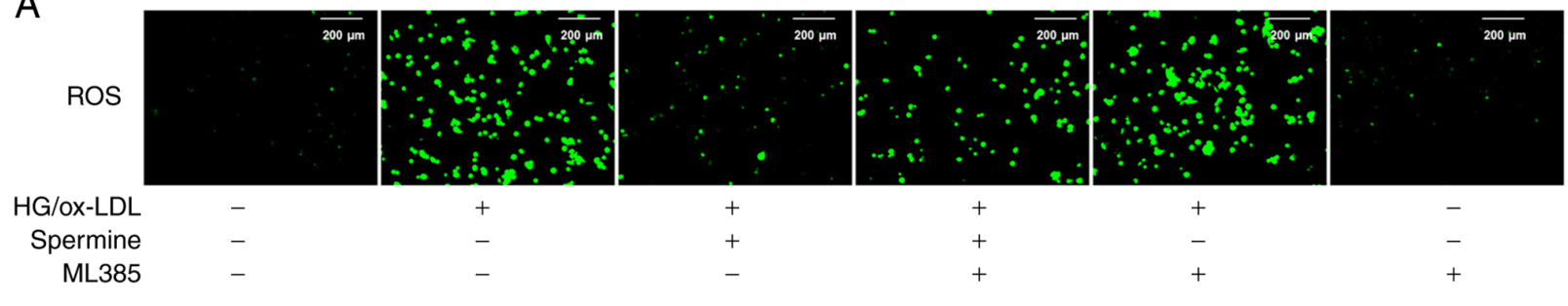

B

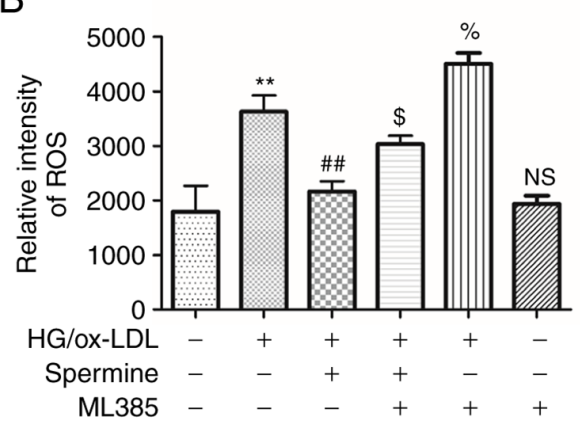

C

D

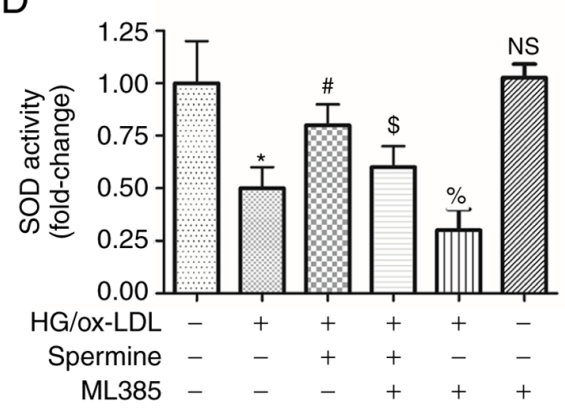

F

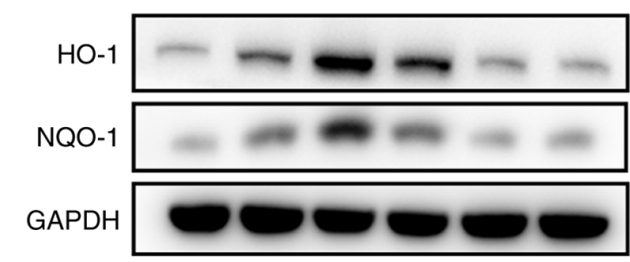

HG/ox-LDL $\quad+\quad+\quad+\quad+\quad+$

Spermine - $-+\quad+\quad-$

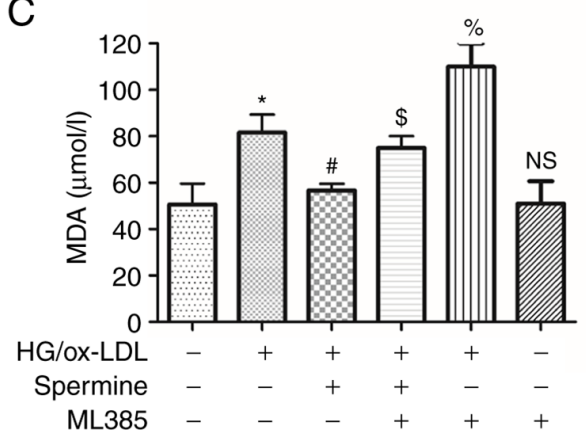

E

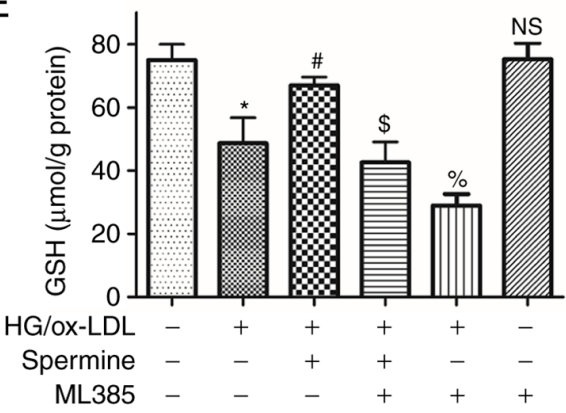

G

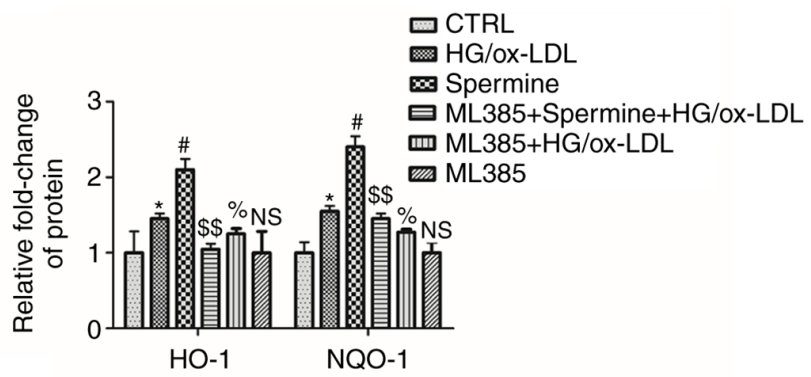

Figure 4. Spermine improves oxidative stress in macrophages exposed to HG/ox-LDL independent of Nrf2. Macrophages were divided into six groups, control group (PBS), HG/ox-LDL group (25 mmol/1 D-Glu and $100 \mu \mathrm{g} / \mathrm{ml}$ ox-LDL treatment for $24 \mathrm{~h})$ and Spermine group (5 $\mu \mathrm{M}$ spermine pretreatment for $30 \mathrm{~min}$ followed by the addition of $25 \mathrm{mmol} / 1 \mathrm{D}-\mathrm{Glu}$ and $100 \mu \mathrm{g} / \mathrm{ml}$ ox-LDL treatment for $24 \mathrm{~h})$, Spermien+ML385+HG/ox-LDL group (5 $\mu \mathrm{M}$ spermine and ML385 pretreatment for $30 \mathrm{~min}$ followed by the addition of $25 \mathrm{mmol} / \mathrm{l} \mathrm{D}-\mathrm{Glu}$ and $100 \mu \mathrm{g} / \mathrm{ml}$ ox-LDL treatment for $24 \mathrm{~h}$ ), ML385 group (5 $\mu \mathrm{M}$ ML385) and ML385+HG/ox-LDL group ( $5 \mu \mathrm{M}$ ML385 and $5 \mathrm{mmol} / \mathrm{l} \mathrm{D}-\mathrm{Glu}$ and $100 \mu \mathrm{g} / \mathrm{ml}$ ox-LDL treatment for $24 \mathrm{~h})$. DCFH-DA was used to evaluated ROS level. GSH, SOD and MDA levels in cell lysates were detected. HO-1 and NQO-1 level in lysates was analysis by western blotting. (A and B) Fluorescence microscopy showed the total ROS level. (C) MDA level. (D) SOD activity. (E) GSH level. (F and G) Protein level of HO-1 and NQO-1. *P<0.05, ** P<0.01 compared with the control group; ${ }^{\#} \mathrm{P}<0.05,{ }^{\# \#} \mathrm{P}<0.01$ compared with the HG/ox-LDL group. ${ }^{\$} \mathrm{P}<0.05,{ }^{\$ \$} \mathrm{P}<0.01$ compared with the spermine group. ${ }^{\%} \mathrm{P}<0.05$ compared with the HG/ox-LDL group. HG, high glucose; ox-LDL, oxidized low-density lipoprotein; Nrf2, nuclear factor erythroid 2-related factor 2; D-Glu, D-glucose; DCFH-DA. 2',7'-Dichlorodihydrofluorescein diacetate; ROS, reactive oxygen species; GSH, glutathione; SOD, superoxide dismutase; MDA, malondialdehyde; NQO-1, NADPH quinone oxidoreductase-1; NS, no statistical significance.

In addition, inhibition of NLRP3 inflammasome activation by spermine was alleviated in the ML385 group compared with the groups with HG/ox-LDL treatment alone (Fig. 5E and F). Collectively, these results indicate that the Nrf2 signaling pathway contributes to the protective effect of spermine against HG/ox-LDL injury in macrophages.

\section{Discussion}

In diabetic atherosclerosis, hyperglycemia and lipids contribute to macrophage dysfunction, which exacerbates inflammatory responses and increases plaque instability $(25,26)$. The present study recreated an environment 
A

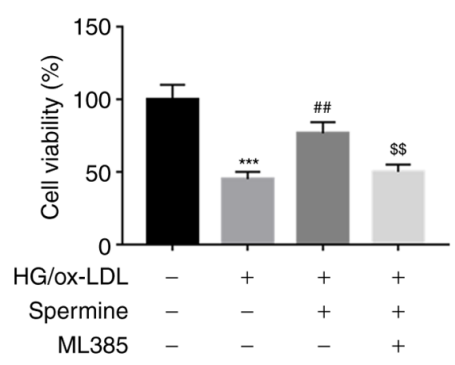

D

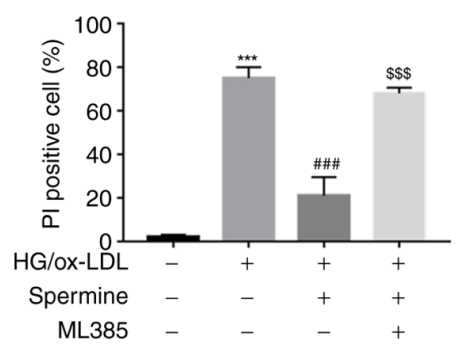

B

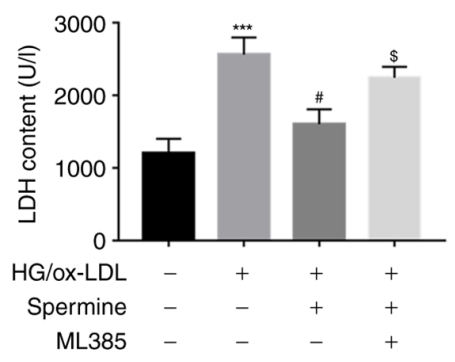

E

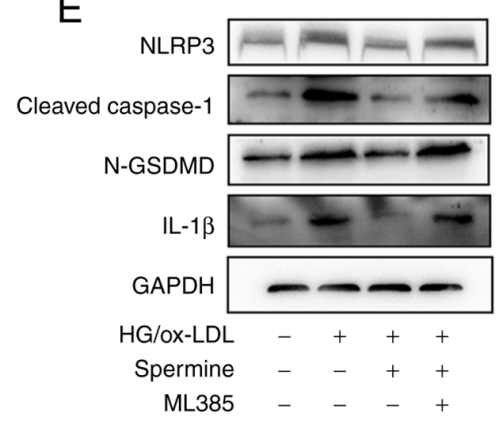

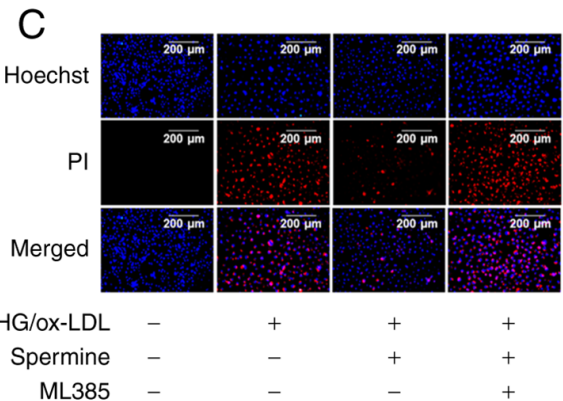

F

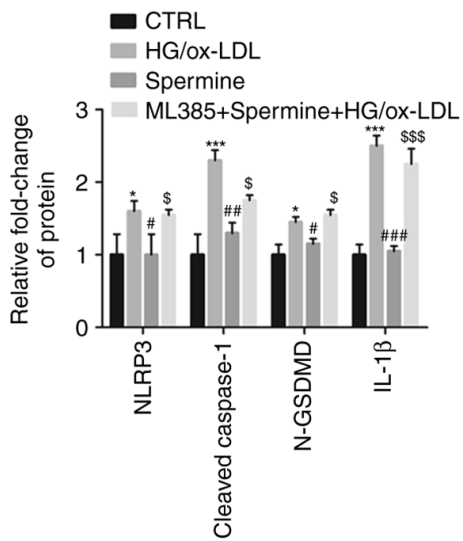

Figure 5. Spermine inhibits macrophage pyroptosis by activating the Nrf2 pathway. Macrophages were divided into three groups, control group (PBS), HG/ox-LDL group (25 mmol/1 D-Glu and $100 \mu \mathrm{g} / \mathrm{ml}$ ox-LDL treatment for $24 \mathrm{~h})$ and Spermine group (5 $\mu \mathrm{M}$ spermine pretreatment for $30 \mathrm{~min}$ followed by the addition of $25 \mathrm{mmol} / 1 \mathrm{D}-\mathrm{Glu}$ and $100 \mu \mathrm{g} / \mathrm{ml}$ ox-LDL treatment for $24 \mathrm{~h}$ ), ML385 group (5 $\mu \mathrm{M}$ spermine and ML385 pretreatment for $30 \mathrm{~min}$ followed by the addition of $25 \mathrm{mmol} / 1 \mathrm{D}-\mathrm{Glu}$ and $100 \mu \mathrm{g} / \mathrm{ml}$ ox-LDL treatment for $24 \mathrm{~h}$ ). CCK-8 assay was used to detect cell viability and LDH kit was used to detect LDH level in cell supernatant. Hoechst/PI staining kit was used to detect cell membrane continuity. Western blotting was used to detected NLRP3, cle-caspase-1, N-GSDMD and IL-1 $\beta$ levels. (A) CCK-8 assay. (B) LDH level in cell supernatant. (C and D) Hoechst/PI was used to detect cell membrane permeability. (E) Protein level of NLRP3, cleaved-caspase-1, IL-1 $\beta$ and N-GSDMD in control, HG/ox-LDL, spermine and ML385 group. (F) Quantification of NLRP3, caspase-1, cleaved-caspase-1, IL-1 $\beta$ and N-GSDMD. ${ }^{*} \mathrm{P}<0.05,{ }^{* * *} \mathrm{P}<0.001$ compared with the control group; ${ }^{\#} \mathrm{P}<0.05,{ }^{\# \#} \mathrm{P}<0.01,{ }^{\# \# \#} \mathrm{P}<0.001$ compared with the HG/ox-LDL group. ${ }^{\$} \mathrm{P}<0.05,{ }^{\$ \$} \mathrm{P}<0.01,{ }^{\$ \$} \mathrm{P}<0.001$ compared with the spermine group. Nrf2, nuclear factor erythroid 2-related factor 2; HG, high glucose; ox-LDL, oxidized low-density lipoprotein; D-Glu, D-glucose; LDH, lactate dehydrogenase; NLRP3, NLR family pyrin domain containing 3; GSDMD, gasdermin D.

similar to that in patients with diabetic atherosclerosis in vitro using HG/ox-LDL conditions. The current study investigated the protective effects of spermine against HG/ox-LDL-induced oxidative stress and pyroptosis in THP-1-derived macrophages. It also investigated the mechanisms underlying this protection. The findings revealed that spermine suppressed HG/ox-LDL-induced macrophage pyroptosis by inhibiting NLRP3 inflammasome activation. In addition, the Nrf2 signaling pathway mediated the protective effects of spermine against HG/ox-LDL injury and oxidative stress and contributed to the inhibitory effect of spermine on HG/ox-LDL-induced NLRP3 inflammasome-mediated pyroptosis in macrophages. These results indicated that spermine attenuated HG/ox-LDL injury in macrophages by inhibiting NLRP3 inflammasome-mediated pyroptosis via activation of the Nrf2 signaling pathway. This provided new insight into the functional role of spermine in diabetic atherosclerosis.

Pyroptosis is a newly discovered proinflammatory mode of programmed cell death and there is abundant evidence that pyroptosis promotes the progression of atherosclerosis (26). In addition, various natural compounds, such as melatonin, colchicine and oxymatrine, have been reported to exert anti-atherogenic effects (27). In addition, Han et al (6) found that sinapic acid attenuated diabetic atherosclerosis by inhibiting bone marrow-derived macrophage pyroptosis in a rat model. Spermine, a natural product of cellular metabolism, has been shown to possess anti-inflammatory and antioxidant properties (28). However, the specific effects of spermine on macrophages remain to be elucidated. The present study found that spermine prevented loss of cell viability usually induced by HG and ox-LDL stimulation. Furthermore, the LDH levels in the supernatant and percentage of PI-positive cells were both significantly decreased in the spermine group compared with those in the HG/ox-LDL group, suggesting spermine increased cell viability and reduced cell membrane disruptions caused by HG/ox-LDL. Western blot analysis indicated that the expression of NLRP3, cleaved caspase-1 and N-GSDMD were also decreased by spermine pretreatment. Furthermore, the levels of IL-1 $\beta$ in the culture supernatants and cell lysates were decreased by spermine treatment. Accordingly, the results suggested that spermine could inhibit macrophage pyroptosis induced by exposure to HG/ox-LDL. It should be noted that in the present study, only PCR and western blotting were used to detect the changes of pyroptosis related genes and proteins in macrophages and flow cytometry was not used to detect the levels of these proteins. This is a limitation of the current study. 
Oxidative stress has been shown to contribute to the activation of NLRP3 inflammation and pyroptosis (29). It was previously demonstrated that ROS enhance the assembly of NLRP3, ASC and caspase-1, which lead to the mutation of cleaved caspase-1 (30). Furthermore, it is observed that ox-LDL induced endothelial pyroptosis by causing ROS overproduction (31). In the present study, it was also found that HG/ox-LDL increased ROS and MDA levels in macrophages and inhibited expression of antioxidant proteins. These effects were reversed by spermine treatment. These data suggested that spermine attenuated macrophage pyroptosis by inhibiting ROS overproduction.

$\mathrm{Nrf} 2$ is a common transcription factor that regulates the transcription of anti-inflammatory and antioxidant proteins, including HO-1, NQO-1, SOD and GSH. The activation of Nrf2, enhanced by luteolin and oxymatrine, suppresses endothelial cell and macrophage pyroptosis (32). Notably, several reports demonstrate that spermine effectively activates $\mathrm{Nrf} 2$ in a range of cell types $(21,33)$. Spermine is also regarded as a ROS scavenger and is capable of protecting DNA from free radical attack (34). However, it is unclear whether spermine has antioxidant effects and whether it activates Nrf2 in HG/ox-LDL-induced pyroptotic macrophages. The current study found that spermine pretreatment decreased ROS and MDA levels, increased SOD and GSH levels and enhanced Nrf2 nuclear translocation. All these effects were reversed by ML385 pretreatment. To test the hypothesis that spermine inhibits macrophage pyroptosis by activating Nrf2 and decreasing oxidative stress, the experimental groups of cells were pretreated with ML385 and then pyroptosis levels and NLRP3 inflammasome protein expression were measured. ML385 attenuated the anti-pyroptosis activity of spermine, causing decreased cell viability and increased levels of NLRP3, cleaved caspase-1 and N-GSDMD. These findings support the hypothesis that spermine attenuates macrophage pyroptosis by activating the Nrf2 pathway.

The biological activity of spermine, which is a common polyamine, has been extensively studied. Spermine has been shown to protect against cardiovascular diseases, including diabetic cardiomyopathy and pulmonary hypertension $(21,35)$. However, to the best of the authors' knowledge, there has been no direct evidence that spermine protects against diabetic atherosclerosis. In the current study, HG/ox-LDL exposure led to macrophage pyroptosis and oxidative stress, which are crucial mechanisms involved in the progression of advanced diabetic atherosclerosis. It was observed that spermine pretreatment significantly increased macrophage viability and suppressed ROS production under HG/ox-LDL conditions. In conclusion, the present findings demonstrated that spermine was protective against macrophage pyroptosis. Notably, the current study provided new evidence that spermine may be a potential drug for the treatment of diabetic atherosclerosis.

\section{Acknowledgements}

Not applicable.

\section{Funding}

The present study was supported by the National Natural Science Foundation of China (grant no. 81770820).

\section{Availability of data and materials}

The datasets used and/or analyzed during the current study are available from the corresponding author on reasonable request.

\section{Authors' contributions}

YL was responsible for the conception and design of the study. YQ, JL and XG aquired and analyzed the data. YQ, LX and LL were involved in drafting the manuscript, the interpretation of the data and revising the manuscript. All authors have read and approved the final manuscript. YL and YQ confirm the authenticity of all the raw data.

\section{Ethics approval and consent to participate}

Not applicable.

\section{Patient consent for publication}

Not applicable.

\section{Competing interests}

The authors declare that they have no competing interests.

\section{References}

1. Soehnlein $\mathrm{O}$ and Libby P: Targeting inflammation in atherosclerosis-from experimental insights to the clinic. Nat Rev Drug Discov 20: 589-610, 2021.

2. Chen X, Zhang D, Li Y, Wang W, Bei W and Guo J: NLRP3 inflammasome and IL-1 $\beta$ pathway in type 2 diabetes and atherosclerosis: Friend or foe? Pharmacol Re 173: 105885, 2021;

3. Senior PA: Glucose as a modifiable cause of atherosclerotic cardiovascular disease: Insights from type 1 diabetes and transplantation. Atherosclerosis 335: 16-22, 2021.

4. Poznyak AV, Nikiforov NG, Starodubova AV, Popkova TV and Orekhov AN: Macrophages and foam cells: Brief overview of their role, linkage, and targeting potential in atherosclerosis. Biomedicines 9: 1221, 2021.

5. Tall AR and Westerterp M: Inflammasomes, neutrophil extracellular traps, and cholesterol. J Lipid Res 60: 721-727, 2019.

6. Han Y, Qiu H, Pei X, Fan Y, Tian H and Geng J: Low-dose sinapic acid abates the pyroptosis of macrophages by downregulation of lncRNA-MALAT1 in rats with diabetic atherosclerosis. J Cardiovasc Pharmacol 71: 104-112, 2018.

7. Zhang C, Song JW, Huang HH, Fan X, Huang L, Deng JN, Tu B, Wang K, Li J, Zhou MJ, et al: NLRP3 inflammasome induces $\mathrm{CD}^{+} \mathrm{T}$ cell loss in chronically HIV-1-infected patients. J Clin Invest 131: e138861, 2021.

8. Zhou Z, He H, Wang K, Shi X, Wang Y, Su Y, Wang Y, Li D, Liu W, Zhang Y, et al: Granzyme a from cytotoxic lymphocytes cleaves GSDMB to trigger pyroptosis in target cells. Science (New York, N.Y.) 368: eaaz7548, 2020

9. Wang Y, Fang C, Xu L, Yang B, Song E and Song Y: Polybrominated diphenyl ether quinone exposure induces atherosclerosis progression via CD36-mediated lipid accumulation, NLRP3 inflammasome activation, and pyroptosis. Chem Res Toxicol 34: 2125-2134, 2021

10. Chen S, Markman JL, Shimada K, Crother TR, Lane M, Abolhesn A, Shah PK and Arditi M: Sex-specific effects of the Nlrp3 inflammasome on atherogenesis in LDL receptor-deficient mice. JACC Basic Transl Sci 5: 582-598, 2020

11. Yang $\mathrm{H}, \mathrm{Lv} \mathrm{H}, \mathrm{Li} \mathrm{H}, \mathrm{Ci} \mathrm{X}$ and Peng L: Oridonin protects LPS-induced acute lung injury by modulating Nrf2-mediated oxidative stress and Nrf2-independent NLRP3 and NF- $\kappa \mathrm{B}$ pathways. Cell Commun Signal 17: 62, 2019 
12. Jayasuriya R, Dhamodharan U, Ali D, Ganesan K, Xu B and Ramkumar KM: Targeting Nrf2/Keap1 signaling pathway by bioactive natural agents: Possible therapeutic strategy to combat liver disease. Phytomedicine 92: 153755, 2021.

13. Wiegman CH, Li F, Ryffel B, Togbe D and Chung KF: Oxidative stress in ozone-induced chronic lung inflammation and emphysema: A facet of chronic obstructive pulmonary disease. Front Immunol 11: 1957, 2020

14. Zhang X, Ding M, Zhu P, Huang H, Zhuang Q, Shen J, Cai Y, Zhao $\mathrm{M}$ and He Q: New insights into the Nrf-2/HO-1 signaling axis and its application in pediatric respiratory diseases. Oxid Med Cell Longev 2019: 3214196, 2019.

15. Xie L, Gu Y, Wen M, Zhao S, Wang W, Ma Y, Meng G, Han Y, Wang Y, Liu G, et al: Hydrogen sulfide induces keap S-sulfhydration and suppresses diabetes-accelerated atherosclerosis via Nrf2 activation. Diabetes 65: 3171-3184, 2016.

16. Liu Y, Yang X, Liu Y, Jiang T, Ren S, Chen J, Xiong H, Yuan M, Li W, Machens HG and Chen Z: NRF2 signalling pathway: New insights and progress in the field of wound healing. J Cell Mol Med 25: 5857-5868, 2021.

17. Alonso-Piñeiro JA, Gonzalez-Rovira A, Sánchez-Gomar I, Moreno JA and Durán-Ruiz MC: Nrf2 and heme oxygenase-1 involvement in atherosclerosis related oxidative stress. Antioxidants (Basel) 10: 1463, 2021

18. Grancara S, Martinis P, Manente S, García-Argáez AN, Tempera G, Bragadin M, Via LD, Agostinelli E and Toninello A: Bidirectional fluxes of spermine across the mitochondrial membrane. Amino Acids 46: 671-679, 2014

19. Li QZ,Zuo ZW, Zhou ZR and Ji Y: Polyamine homeostasis-based strategies for cancer: The role of combination regimens. Eur J Pharmacol 910: 174456, 2021.

20. Matsumoto M: Prevention of atherosclerosis by the induction of microbial polyamine production in the intestinal lumen. Biol Pharm Bull 43: 221-229, 2020.

21. Wang Y, Chen J, Li S, Zhang X, Guo Z, Hu J, Shao X, Song N, Zhao Y, Li H, et al: Exogenous spermine attenuates rat diabetic cardiomyopathy via suppressing ROS-p53 mediated downregulation of calcium-sensitive receptor. Redox Biol 32: 101514, 2020.

22. Zhao S, Song T, Gu Y, Zhang Y, Cao S, Miao Q, Zhang X, Chen H, Gao Y, Zhang L, et al: Hydrogen sulfide alleviates liver injury through the S-sulfhydrated-kelch-like ECH-associated protein $1 /$ nuclear erythroid 2 -related factor $2 /$ low-density lipoprotein receptor-related protein 1 pathway. Hepatology 73 : 282-302, 2021

23. Verreth W, De Keyzer D, Davey PC, Geeraert B, Mertens A, Herregods MC, Smith G, Desjardins F, Balligand JL and Holvoet P: Rosuvastatin restores superoxide dismutase expression and inhibits accumulation of oxidized LDL in the aortic arch of obese dyslipidemic mice. Br J Pharmacol 151: 347-355, 2007

24. Qiu Z, He Y, Ming H, Lei S, Leng Y and Xia ZY: Lipopolysaccharide (LPS) aggravates high glucose- and hypoxia/reoxygenation-induced injury through activating ROS-dependent NLRP3 inflammasome-mediated pyroptosis in H9C2 cardiomyocytes. J Diabetes Res 2019: 8151836, 2019.
25. Josefs T, Barrett TJ, Brown EJ, Quezada A, Wu X, Voisin M, Amengual $J$ and Fisher EA: Neutrophil extracellular traps promote macrophage inflammation and impair atherosclerosis resolution in diabetic mice. JCI insight 5: e134796, 2020.

26. Li P, Wang Y, Liu X, Liu B, Wang ZY, Xie F, Qiao W, Liang ES, Lu QH and Zhang MX: Loss of PARP-1 attenuates diabetic arteriosclerotic calcification via Stat1/Runx2 axis. Cell Death Dis 11: 22, 2020.

27. Moss JW and Ramji DP: Nutraceutical therapies for atherosclerosis. Nat Rev Cardiol 13: 513-532, 2016.

28. Igarashi $\mathrm{K}$ and Kashiwagi K: Functional roles of polyamines and their metabolite acrolein in eukaryotic cells. Amino Acids 53: 1473-1492, 2021

29. Lu J, Xu L, Zeng Z, Xue C, Li J, Chen X, Zhou P, Lin S, Liao Y, $\mathrm{Du} \mathrm{X}$, et al: Normothermic ex vivo heart perfusion combined with melatonin enhances myocardial protection in rat donation after circulatory death hearts via inhibiting NLRP3 inflammasome-mediated pyroptosis. Front Cell Dev Biol 9: 733183, 2021.

30. Abderrazak A, Syrovets T, Couchie D, El Hadri K, Friguet B, Simmet T and Rouis M: NLRP3 inflammasome: From a danger signal sensor to a regulatory node of oxidative stress and inflammatory diseases. Redox Biol 4: 296-307, 2015.

31. Chen JJ, Tao J, Zhang XL, Xia LZ, Zeng JF, Zhang H, Wei DH, Lv YC, Li GH and Wang Z: Inhibition of the ox-LDL-induced pyroptosis by FGF21 of human umbilical vein endothelial cells through the TET2-UQCRC1-ROS pathway. DNA Cell Biol 39: 661-670, 2020

32. Zou Y, Luo X, Feng Y, Fang S, Tian J, Yu B and Li J: Luteolin prevents THP-1 macrophage pyroptosis by suppressing ROS production via Nrf2 activation. Chem Biol Interact 345: 109573, 2021.

33. Wang J, Li S, Wang J, Wu F, Chen Y, Zhang H, Guo Y, Lin Y, Li L, $\mathrm{Yu}$ X, et al: Spermidine alleviates cardiac aging by improving mitochondrial biogenesis and function. Aging (Albany NY) 12: 650-671, 2020.

34. Mohammadi M, Aelaei M and Saidi M: Pre-harvest spray of GABA and spermine delays postharvest senescence and alleviates chilling injury of gerbera cut flowers during cold storage. Sci Rep 11: 14166, 2021

35. He YY, Yan Y, Jiang X, Zhao JH, Wang Z, Wu T, Wang Y, Guo SS, Ye J, Lian TY, et al: Spermine promotes pulmonary vascular remodelling and its synthase is a therapeutic target for pulmonary arterial hypertension. Eur Respir J 56: 2000522 , 2020.

This work is licensed under a Creative Commons Attribution-NonCommercial-NoDerivatives 4.0 International (CC BY-NC-ND 4.0) License. 